\title{
Functionalized Silicate Nanochannels: Towards Applications in Drug Delivery and Solar Energy Conversion
}

\author{
Dominik Brühwiler*, Hanna Ritter, Jan Hinrich Ramm, Le-Quyenh Dieu, Christophe Bauer, \\ Igor Dolamic, and Nando Gartmann
}

\begin{abstract}
The defined pore networks of silicate-based molecular sieves are attractive for the development of highly organized inorganic/organic hybrid materials. Efficient energy transfer systems are obtained upon inclusion of dye molecules into the one-dimensional channels of zeolite $\mathrm{L}$. The resulting host-guest materials form the basis for an advanced luminescent solar concentrator. The selective functionalization of the channel entrances with zinc phthalocyanine offers further possibilities in terms of employing the dye-zeolite composites for the sensitization of organic solar cells. Having larger pore sizes than zeolites as well as highly modifiable channel walls, mesoporous silicas have recently emerged as versatile starting materials for the synthesis of drug delivery devices. One of the most challenging aspects in this context is the control and the analysis of the functional group distribution. This topic is discussed on the basis of our recent work on the reaction of aminopropylalkoxysilanes with mesoporous silica MCM-41.
\end{abstract}

Keywords: Drug delivery $\cdot$ Luminescent solar concentrator $\cdot$ Mesoporous silica $\cdot$ Zeolite

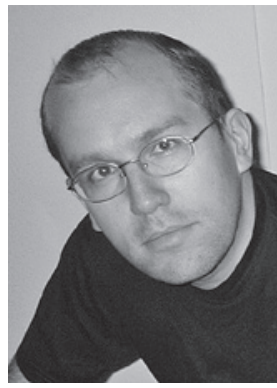

Dominik Brühwiler received his $\mathrm{PhD}$ from the University of Bern in 2001, followed by a postdoctoral fellowship at the Lawrence Berkeley National Laboratory. Returning to Bern, he worked as a Young Researcher in the European Research Training Network (RTN) 'Nanochannel'. In December 2004, he took a position as an Oberassistent at the University of Zürich and is currently leading a research group specializing in the synthesis, functionalization, and organization of microporous and mesoporous silicates. $\mathrm{He}$ is a member of the steering committee of the Marie Curie RTN 'Nanomatch'.

${ }^{\star}$ Correspondence: Dr. D. Brühwiler

University of Zürich

Institute of Inorganic Chemistry

Winterthurerstrasse 190

$\mathrm{CH}-8057$ Zürich

Tel.: +4144635 4630

Fax: + 41446356802

E-mail: bruehwi@aci.uzh.ch

\section{Introduction}

The unique properties of the defined channel systems of silicate-based porous materials, such as zeolites or mesoporous silica, are of interest for a variety of applications, including fields as diverse as catalysis, ${ }^{[1]}$ biological imaging, ${ }^{[2]}$ and drug delivery. ${ }^{[3,4]}$ Appropriate functionalization of the porous host materials is the key to a successful implementation. Extending the organization provided by a given channel system to a macroscopic level and/or establishing an interaction with the surrounding medium or external objects requires multiple degrees of modification. This aspect of the chemistry of microporous and mesoporous materials is discussed in the following by two examples of our recent research. In the case of mesoporous silica, we will focus on the synthetic and analytical challenges associated with the controlled placement of functional groups on specific parts of the external and internal surfaces, particularly concerning potential applications of the materials as drug delivery devices. The possibilities in terms of achieving supramolecular organization of guest molecules in the channel systems of zeolites are illustrated by our research on dye-zeolite energy transfer materials. These highly organized composites can be used as color changing media, photonic antenna systems for the sensitization of organic solar cells, or light-absorbing and emitting components in luminescent solar concentrators. [5]

\section{Mesoporous Silica and Zeolite L}

Since the first report of the M41S type materials in 1992, ${ }^{[6]}$ the synthesis of mesoporous silica has been further developed to provide a wide range of pore sizes and morphologies (spheres, rods, fibers, thin films, etc.). ${ }^{[7]}$ Narrow pore size distributions (PSDs) are obtained by means of a structure-directing agent (SDA), such as alkyltrimethylammonium ions ${ }^{[6]}$ or block copolymers (Fig. 1). ${ }^{[8]}$ The SDA is removed after the formation of the mesoporous framework by calcination at elevated temperatures (typically around $500{ }^{\circ} \mathrm{C}$ ) or extraction. Functional groups can be introduced by postsynthetic grafting or cocondensation. ${ }^{[9]}$ The latter method implies that the moiety to be introduced is stable under the conditions of the mesoporous silica synthesis. While most procedures for the synthesis of mesoporous silica require the use of hydrothermal conditions, high-quality materials can also be prepared by means of scale-up friendly room temperature syntheses and without necessitating costly lab equipment. ${ }^{[10]}$ Fig. 1 shows the PSDs of mesoporous silica MCM-41 synthesized under hydrothermal conditions ${ }^{[11]}$ in comparison to the corresponding materials prepared at room temperature.

Zeolites are classified as microporous materials (pore sizes smaller than $2 \mathrm{~nm}$, by IUPAC definition). [12] Unlike their mesoporous counterparts that feature amorphous pore walls, zeolites are crystalline silicates or aluminosilicates. Our work has 


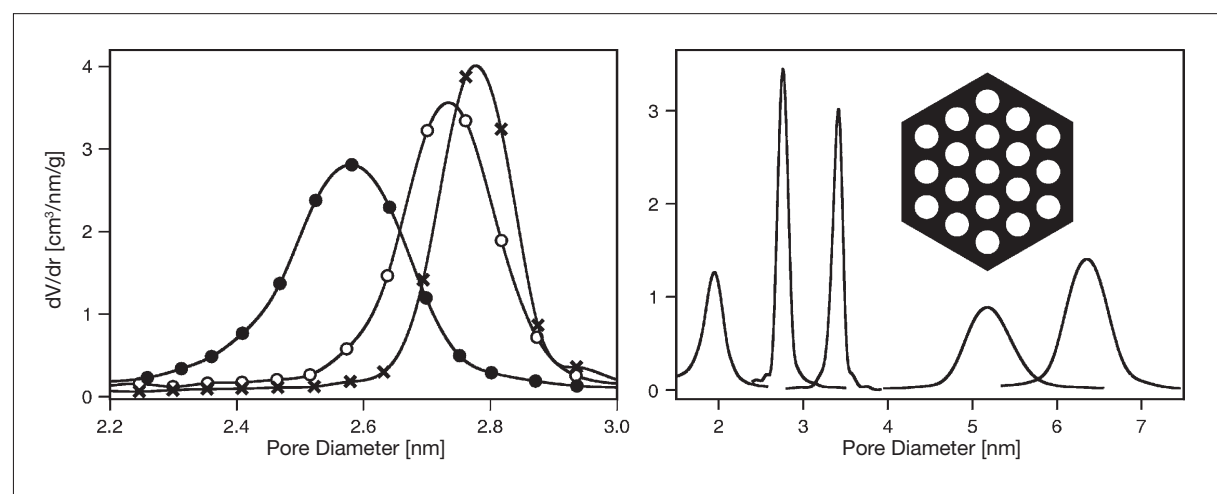

Fig. 1. Left: PSD of mesoporous silica MCM- 41 synthesized at $110^{\circ} \mathrm{C}$ under autogenous pressure and subsequent calcination at $550^{\circ} \mathrm{C}$ (crosses), compared to materials prepared by the same procedure but conducting the condensation step at room temperature with removal of the SDA by calcination (solid circles) or extraction (empty circles). Right: PSD of five different mesoporous silica materials. The samples with pore sizes below $4 \mathrm{~nm}$ are of the MCM-41 type, whereas the slightly broader distributions above $4 \mathrm{~nm}$ have been achieved with SBA-15 type materials. All materials feature a 2D hexagonal structure. The MCM- 41 materials have thin channel walls (approximately $0.8 \mathrm{~nm}$ ), whereas SBA-15 type materials are characterized by thicker channel walls. PSDs were calculated from the desorption branch of the nitrogen isotherm by the BarrettJoyner-Halenda (BJH) method.

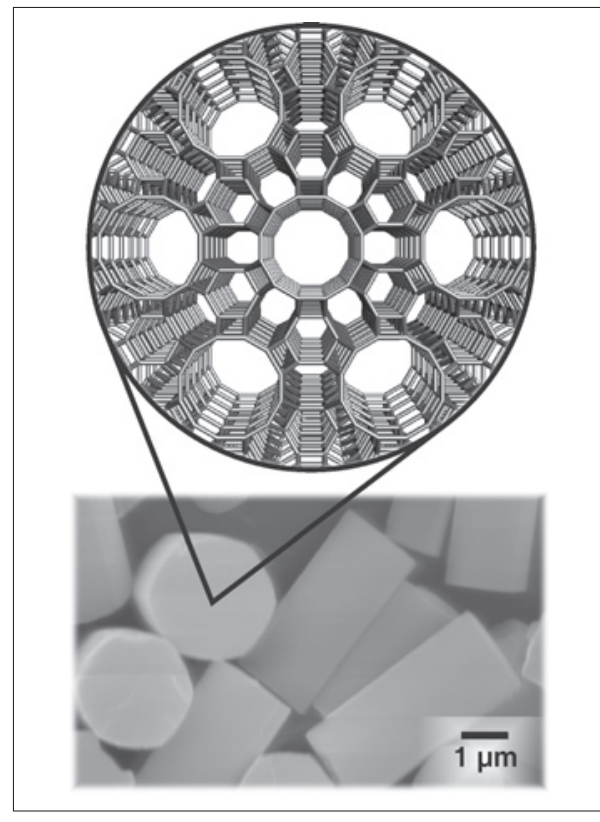

Fig. 2. Scanning electron microscopy (SEM) image of rod-shaped zeolite $L$ crystals. The detail shows the hexagonal framework viewed along the cylinder axis. A crystal with a diameter of $2 \mu \mathrm{m}$ contains approximately $10^{6}$ parallel channels.

mainly focused on zeolite L. Similar to the mesoporous MCM-41, zeolite L is characterized by a hexagonal array of parallel one-dimensional channels. The free diameter of the channels varies from $0.71 \mathrm{~nm}$ (narrowest part) to $1.26 \mathrm{~nm}$ (widest part). Crystals of zeolite L are usually obtained with cylindrical morphology. The parallel main channels run through the entire crystal along the cylinder axis and are accessible from the base surfaces (Fig. 2). By careful control of the synthesis parameters, the aspect ratio of the cylindrical crystals can be adjusted to afford rod-shaped and even disc-shaped particles. ${ }^{[13]}$ The latter are ideal for the preparation of oriented layers.

Apart from the pore size, a key difference between mesoporous silica and zeolite lies in the nature of the pore surface. The pore surface of mesoporous silica is characterized by a large number of silanol groups (approximately three silanols per $\mathrm{nm}^{2}$, depending on the mesoporous silica type and on the thermal treatment during synthesis and calcination/extraction). ${ }^{[14]}$ In zeolites, on the other hand, silanol groups are predominantly found on the external surface and to a lesser extent at defect sites in the pores where the framework is interrupted.

\section{Functionalization of Mesoporous Silica Surfaces}

\subsection{Drug Delivery Devices Based on Mesoporous Silica}

Amorphous silica particles are nontoxic and highly biocompatible. The defined, structurally stable one-dimensional channel system of mesoporous silica such as MCM-41 ${ }^{[6]}$ or SBA-15[8] furthermore offers possibilities to control the uptake and release of drug molecules. The optimum size of the mesoporous silica particles for drug delivery mainly depends on the route of administration as well as on the target. For intravenous applications, for example, the particle size should be between 50 and $300 \mathrm{~nm} .{ }^{[15]}$ Functional groups on the external particle surface define the interaction with the surrounding medium. Solving different tasks, such as targeting, avoiding detection by the im- mune system, or preventing particle aggregation, requires multi-functionalization. In addition to the modification of the external surface, the internal (pore) surface needs to be functionalized independently with moieties for the optimization of drug adsorption. An ideal drug delivery system should further be equipped with stimuli-responsive gates ensuring zero release before reaching the target. The concept of opening and closing mesoporous silica channels by an external physical or chemical stimulus has recently gained substantial interest. [3]

\subsection{Synthetic Challenges}

As outlined above, a potential drug delivery device based on mesoporous silica requires different types of covalently attached functional groups, each with a predetermined surface location. The ability to control and analyze the distribution of these groups is crucial. In order to investigate the parameters that affect the surface distribution, we have been mainly focusing on amines, as they are among the most frequently employed groups for the modification of mesoporous silica surfaces. Once the amines are anchored, a further moiety can be coupled by means of amine-reactive derivatives such as isothiocyanates or sulfonylchlorides. We have found this to be especially useful for the analysis of the amino group distribution, as it allows for the attachment of fluorescent labels which can be analyzed by methods based on fluorescence quenching or confocal laser scanning microscopy (CLSM).

Most reactions targeting the anchoring of amino groups rely on 3-aminopropyltrialkoxysilanes (alkoxy $=$ methoxy or ethoxy), which are most commonly deposited from toluene. When a random distribution of the functional groups is desired (in cases of less than full coverage), the use of dry toluene is essential in order to minimize silane cross-linking and prevent the eventual formation of a non-uniform surface coverage. However, even when keeping trace water to a minimum, deposition from toluene produces aggregated grafted amino groups and preferential external surface grafting, as well as anchoring to internal surface sites close to the pore entrances. ${ }^{[16]}$ Interestingly, the choice of solvent determines to a certain extent the distribution of the surface-grafted amino groups. Less aggregation and increased site isolation has for example been obtained upon deposition from alcohols or THF.[17,18] The deposition of alkoxysilanes from the gas phase offers multiple advantages over the common solvent-based techniques: The elimination of trace water and silane oligomers is straightforward, while the absence of a solvent during the deposition 
and washing of the samples is particularly beneficial for ecological and economical reasons. We have recently shown that amino-functionalized MCM-41 prepared by gas phase deposition features a narrow PSD, comparing favorably to corresponding samples prepared by deposition from toluene. ${ }^{[10]}$

The distribution of the amino groups can be tuned by adjusting the reactivity of the respective alkoxysilane precursor. We have investigated the relationship between the reactivity and the surface distribution by depositing submonolayer amounts of various alkoxysilane precursors (Fig. 3) onto MCM-41 from toluene. ${ }^{[18]}$ Dipodal trialkoxysilanes, such as bis(trimethoxysilyl)propylamine (BTMSPA), reacted preferably with sites on the external surface and at the pore entrances, whereas monoalkoxysilanes, such as 3-aminopropyldimethylmethoxysilane (APDMMS), produced a more uniform distribution with excellent site isolation. The commonly employed trialkoxysilanes showed intermediate behavior. Due to the comparatively large average pore diameter $(3.6 \mathrm{~nm})$ and the small amounts of alkoxysilane precursors (leading to a coverage of one amino group per $15 \mathrm{~nm}^{2}$ assuming uniform distribution), the different distributions of the grafted amino groups cannot be attributed exclusively to sterical effects. In fact, the bulky 3-aminopropyldiisopropyltriethoxysilane (APDIPES) produced a more uniform distribution of grafted amino groups than 3-aminopropyltrimethoxysilane (APTMS). The specific surface distributions are rather a result of the subtle interplay between the number of alkoxy groups per molecule and the nature of the amine (primary or secondary). ${ }^{[18]}$

A common strategy to passivate the external surface of mesoporous silica is to

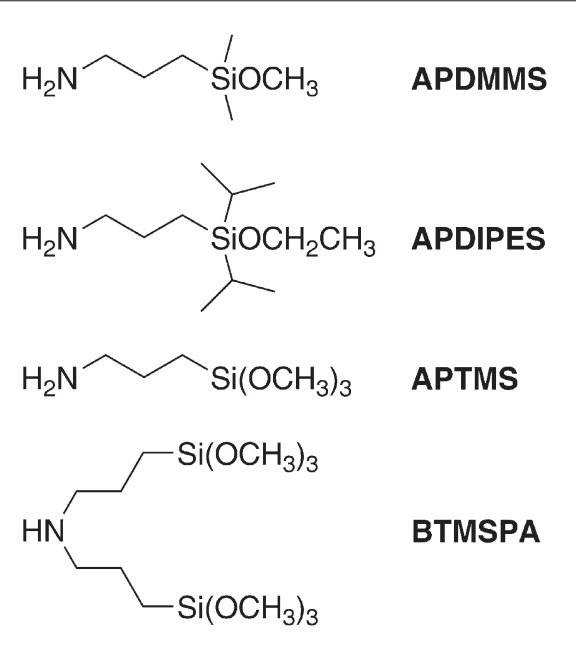

Fig. 3. Structures and abbreviations of selected alkoxysilanes for the functionalization of mesoporous silica with amino groups. employ a small amount of dichlorodiphenylsilane. ${ }^{[19]}$ This method relies on the assumption that the most kinetically available silanol groups are those on the external surface. Quantitative evidence concerning the distribution of the grafted silane is, however, difficult to obtain in this case. We are currently investigating to what extent the relationship of reactivity and surface location can be extended to silanes containing other functional groups.

When developing methods for the selective modification of the external surface, it is tempting to take advantage of the fact that the mesoporous silica is synthesized with a SDA which subsequently blocks the pores unless it is removed by calcination or extraction. Careful examination of functionalization methods employing SDA-filled pores reveals that the selectivity for the external surface is usually not sufficient. The reaction of APTMS with MCM-41 still containing the SDA (hexadecyltrimethylammonium ions) is such an example. The APTMS molecules are apparently able to diffuse into the pores despite the presence of the SDA. An influence on the PSD is already observed at low APTMS loading (Fig. 4). It is reasonable to assume that grafting to the pore surface occurs as a result of an exchange between APTMS and SDA. ${ }^{[20]}$ Our current approach employs bulky protecting groups to enhance the selectivity for external surface grafting. Results from the investigation of pore blocking effects suggest that protecting groups with sizes similar to half

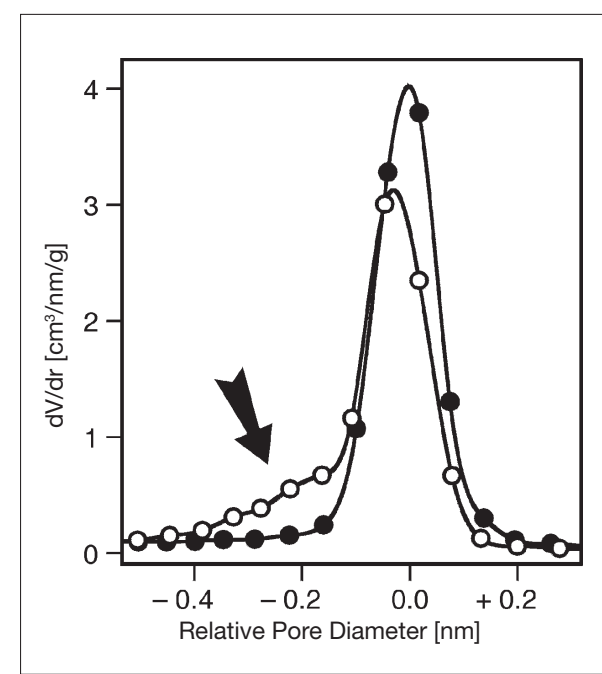

Fig. 4. PSD of MCM-41 reacted with APTMS and subsequent extraction of the SDA (empty circles). A corresponding blind sample is given for comparison (solid circles). APTMS clearly reacts with pore surface sites, leading to a reduction of the pore size. A small amount of APTMS was used in this experiment (corresponding to a surface coverage of 0.1 amino groups per $\mathrm{nm}^{2}$ assuming homogeneous distribution). PSDs were calculated from the desorption branch of the nitrogen isotherm by the Barrett-Joyner-Halenda (BJH) method. the pore size are most likely sufficient to yield a high selectivity for the external surface. ${ }^{[21]}$

\subsection{Analytical Challenges}

The determination of the number of organic functional groups bound to the surface of mesoporous silica is not straightforward. Conventional techniques such as elemental analysis provide reliable results for high surface concentrations, but are generally not applicable in the low loading regime. Chromogenic reactions, as for example the reaction of ninhydrin with surface-grafted amines, are a promising alternative. It is, however, essential to decompose the mesoporous framework before applying such methods, as functional groups deep inside the pores might not be accessible, leading to an analysis result which is biased by the distribution of the functional groups on the silica surface. To avoid this problem, we have developed a procedure based on the fluorogenic aminederivatization reaction of fluorescamine ${ }^{[22]}$ that can be used under the alkaline conditions required for the decomposition of the mesoporous silica framework. A wide range of amino contents can be covered with this analytical tool, allowing a reliable determination of the amount of surfacegrafted amino groups independent of their distribution. ${ }^{[10]}$

To analyze the surface distributions obtained with different aminopropylalkoxysilane precursors (Fig. 3), we labeled the grafted amino groups with fluorescein isothiocyanate (FITC). The results were surprising. Samples containing identical amounts of labeled amino groups featured significantly different PSDs and luminescence intensities (Fig. 5). ${ }^{[18]}$ This enabled us to classify the aminopropylalkoxysilanes according to their tendency to produce uniform distributions on the mesoporous silica surface. A comparatively large average pore diameter of the labeled sample indicates that the functional groups are concentrated on the external particle surface and on the pore surface close to the channel entrances (BTMSPA), whereas a small average pore diameter is obtained as a result of a more homogeneous distribution of the functional groups (APDMMS). Small distances between the FITC-labeled amino groups cause self-quenching of the luminescence, an effect which was particularly pronounced in BTMSPA-modified samples. Uniform distributions, on the other hand, are identified by a high luminescence intensity. Functionalized mesoporous silica particles exhibiting bright luminescence have recently attracted increased attention due to their potential use in intracellular imaging applications. ${ }^{[23]}$ Labeling with pyrene sulfonylchloride (PSC) affords similar 


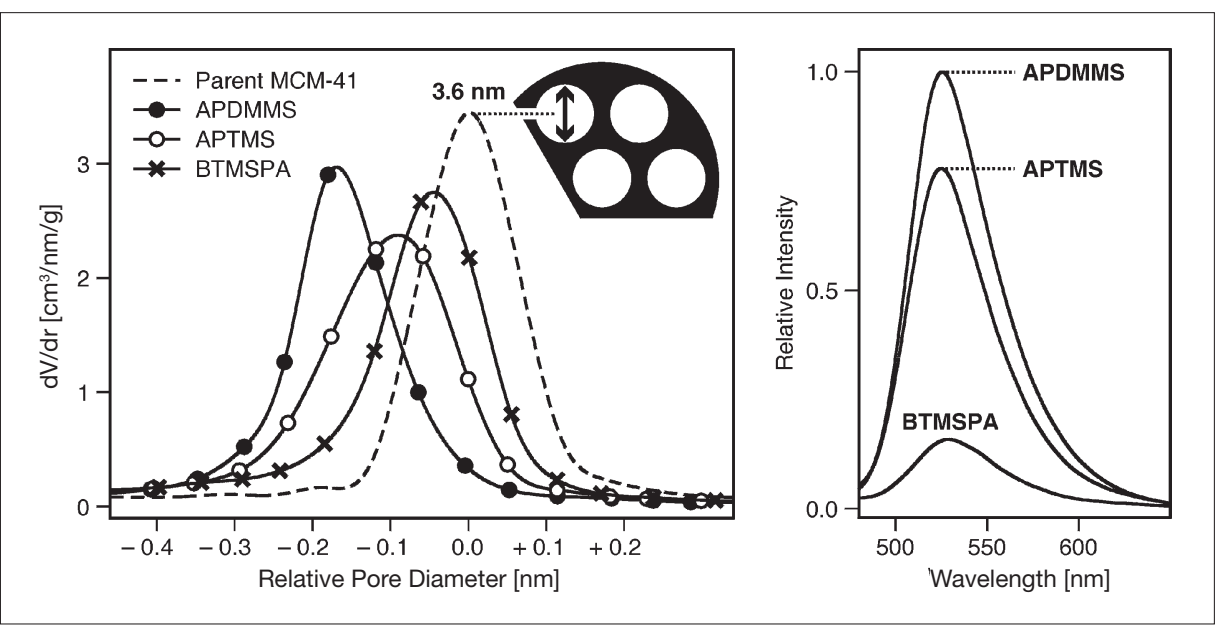

Fig. 5. Left: PSDs of FITC-coupled amino-functionalized samples prepared with different alkoxysilane precursors. Right: Luminescence spectra of the same samples excited at $450 \mathrm{~nm}$. Fluorescein contents are $(20.0 \pm 1.0) \mu \mathrm{mol} / \mathrm{g}$. PSDs were calculated from the desorption branch of the nitrogen isotherm by the Barrett-Joyner-Halenda (BJH) method.

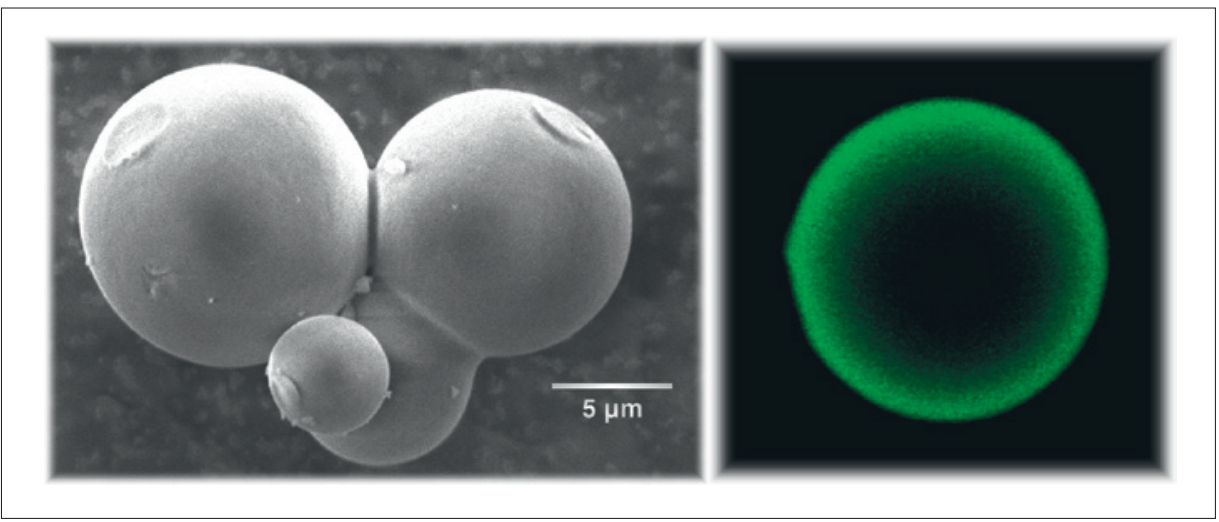

Fig. 6. Left: SEM image of spherical mesoporous silica particles (5.3 nm average pore diameter). Right: CLSM cross-section of a spherical mesoporous silica particle (ca. $8 \mu \mathrm{m}$ diameter) functionalized with amino groups and subsequently labeled with FITC. The dark center of the particle indicates that the amino groups are predominantly located on the external surface and on the pore surface close to the pore entrances. The external surface area accounts for only $2 \%$ of the total surface area. The latter is approximately $800 \mathrm{~m}^{2} / \mathrm{g}$ in this case.

information concerning the proximity of the amino groups. The formation of excimers is indicative of densely spaced PSC-labeled groups. When applying such methods for comparative studies, it is essential that the samples contain identical numbers of labeled groups.

We are currently investigating the potential of CLSM with regard to imaging the functional group distribution. To obtain a reasonable resolution, large particles, preferably in the vicinity of about $5 \mu \mathrm{m}$, with defined morphology are required. The synthesis of such materials is not straightforward, as the majority of the reported mesoporous silica syntheses yield irregular particle morphologies with sizes below $2 \mu \mathrm{m}$. Fig. 6 shows an example of large mesoporous silica spheres and a non-uniform distribution of FITC-labeled amino groups detected by CLSM. In addition to the CLSM analysis, we are developing a method which can also be used for smaller functional groups. We have termed this technique 'surface-selective quenching', as it employs quencher species that are too large to enter the channels of a given mesoporous material and therefore exclusively quench the luminescence of labels located on the external particle surface. featuring a defined distribution of labeled functional groups can then be used to estimate the relative amounts of external and internal grafting.

\section{Dye-Zeolite Composites}

\subsection{Photonic Antenna Systems with Phthalocyanine Stopcocks}

A variety of dye molecules have been introduced into the channels of zeolite $\mathrm{L}$ particles containing fluorescence-labeled The comparison with reference systems by deposition from the gas phase (neutral dyes) or ion exchange from solution (cationic dyes). ${ }^{[24]}$ As sequentially included dyes cannot pass each other in the narrow zeolite L channels, dye-zeolite composites featuring defined dye domains can be synthesized. Due to the strong confinement, high local concentrations of nonaggregated dye molecules are achievable, enabling efficient Förster resonance energy transfer (FRET). The transport of electronic excitation energy along the channels of the dye-zeolite crystals becomes a particularly useful process if one is able to extract the energy and subsequently transfer it to an external object. In order to solve this complex task, the channel entrances of the zeolite crystals need to be functionalized with appropriate acceptor molecules. Due to the relatively narrow channels of zeolite L, methods that rely on steric constraints have provided a high selectivity for the modification of the channel entrances. Molecules that are used for this purpose have the typical shape of a stopcock, consisting of a large (chromophoric) head and a long narrow tail.[24] While the tail is able to enter the channels, the head is too large and remains on the external surface. An example of such a stopcock molecule is shown in Fig. 7.[25] Stopcocks are usually deposited onto the zeolite crystals from a solvent. The optimization of the channel entrance adsorption (with tails extending into the channels) while simultaneously avoiding adsorption on the coat of the crystals is a delicate process that

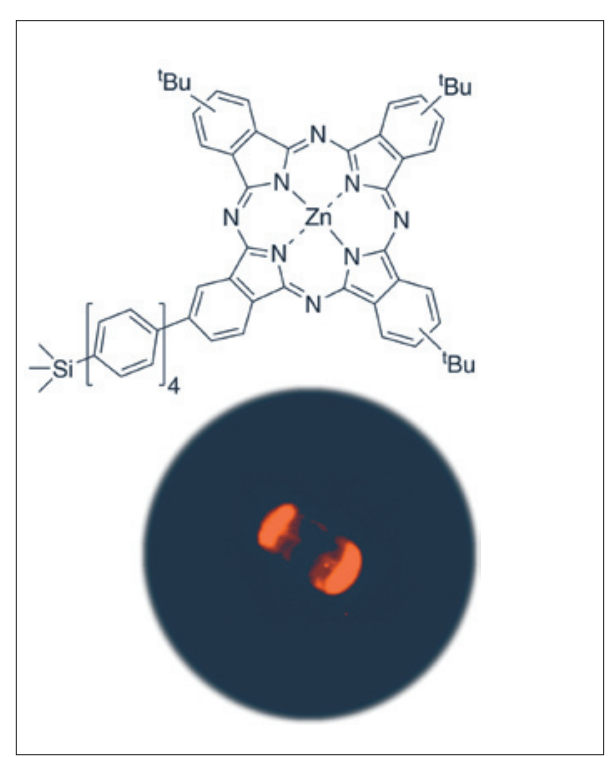

Fig. 7. A stopcock with a zinc phthalocyanine head which can be selectively adsorbed at the channel entrances of zeolite L. The fluorescence microscope image shows a zeolite crystal of ca. $5 \mu \mathrm{m}$ length that has been functionalized with this particular stopcock. Bright fluorescence is observed at the base surfaces of the cylindrical crystal. 
requires extensive screening of different solvents and adsorption conditions. Our work mainly focuses on stopcocks with zinc phthalocyanine heads. ${ }^{[25]}$ These are of special interest for the sensitization of organic solar cells. [26]

\subsection{An Advanced Luminescent Solar Concentrator}

The concept of using luminescent solar concentrators (LSCs) to reduce the cost of photovoltaic power dates back to the late 1970s. ${ }^{[27]}$ Intensive research continued until the early 1980 s, when several problems, outlined below, hindered further development. Recent advances in the area of organic and inorganic luminescent materials have led to a renewed interest in LSCs. ${ }^{[28]}$

A conventional LSC consists of a transparent material (usually polymethylmethacrylate PMMA) that contains luminescent dye molecules (Fig. 8). ${ }^{[27]}$ Photons entering the face of the LSC plate are absorbed by the dye molecules with subsequent emission of photons. A large fraction of these emitted photons are trapped by total internal reflection and travel towards the edges of the plate, where they can be received, for example, by a photovoltaic cell or a thermal absorber. The ratio of the area of the face to the area of the edge corresponds to the geometric gain for the LSC plate. An efficient LSC with a high geometric gain has the potential of significantly lowering the cost of solar energy conversion, as a given area covered with a comparatively expensive solar cell can be reduced to the much smaller edge area of a corresponding LSC plate. Contrary to conventional optical concentrators such as lenses, LSCs are able to concentrate diffuse light and therefore do not require tracking of the sun. Unfortunately, conventional LSCs suffer from a series of major problems. Organic molecules with a high fluorescence quantum yield generally feature a considerable overlap between absorption and emission spectra. This leads to self-absorption, implying that luminescent photons have a high probability of being reabsorbed on their way to the edges of the LSC plate.
A quantum of energy undergoing successive absorption and emission processes basically executes a random walk in the LSC, thereby increasing the probability of being lost, for example by a non-unit fluorescence quantum yield or by emission into the escape cone. ${ }^{[29]}$ The latter process is a significant loss mechanism. For a conventional LSC based on a PMMA matrix, the escape probability amounts to $26 \%$ for isotropic absorption and emission. ${ }^{[30]}$

We are developing an advanced LSC that has the potential to solve the self-absorption problem and reduce the relative amount of photons emitted into the escape cone. ${ }^{[26,31]}$ The concept is based on a thin zeolite-polymer layer. The zeolite contains a large number of donor molecules, which absorb the incident light and subsequently transfer the electronic excitation energy to a comparatively small number of acceptor dyes. As the donor molecules are not able to absorb the acceptor luminescence, photons emitted by the acceptor molecules can travel to the edges of the LSC plate without being reabsorbed. Due to their low concentration, self-absorption by the acceptor dyes is expected to become negligible. The electronic transition dipole moments of the acceptor dyes can further be aligned by orienting the zeolite crystals. This offers possibilities of generating optical anisotropy and reducing the emission into the escape cone.

Organic dyes in conventional LSCs are often derivatives of perylene, having fluorescence quantum efficiencies approaching 1.00, high extinction coefficients, and superior photostability when compared to other dyes with similar photophysical properties.[32] Despite the high intrinsic stability of these dyes, the stability of conventional LSCs is not sufficient for long term (10-20 years) outdoor application. We can use many of the dyes initially developed for conventional LSCs in our advanced LSC concept, as their size permits inclusion into the channels of zeolite L. Unlike the conventional approach of directly dispersing the dye in a polymer, we are not limited by the often low solubility of the perylene pigments. It is expected that the strong confinement in the zeolite channels will be beneficial in terms of the photostability of the dyes and therefore lead to a prolonged lifetime of the LSC devices. Furthermore, the zeolite channels provide an environment that is chemically relatively inert compared to organic polymers, which often contain reactive species due to incomplete polymerization.

\section{Conclusions}

Silicate-based materials with defined nanochannels provide a platform for the development of robust and highly functional particles. Zeolites are ideal hosts for the supramolecular organization of small molecules. Selective functionalization of the channel entrances establishes communication between the included guest molecules and external objects. With pore sizes in the range of 2 to $50 \mathrm{~nm}$, mesoporous materials can accommodate larger guests and additionally provide a high density of binding sites for functional groups. A key to potential applications of functionalized mesoporous silica lies within the ability to control the surface distribution of the functional groups. To develop the corresponding modification techniques, methods accomplishing the reliable analysis of the functional group distribution are indispensable. Fluorescent labeling opens various possibilities for studying the functional group distribution, including quenching experiments and confocal laser scanning microscopy. These methods effectively complement the data obtained from the analysis of the nitrogen sorption isotherms.

\section{Acknowledgments}

Our research is financially supported by the Swiss National Science Foundation (Project 200020-117591), the Swiss Commission of Technology and Innovation (KTI/CTI, Project 9231.2 PFNM-NM), and the European Commission through the Human Potential Programme (Marie-Curie RTN Nanomatch, Grant No. MRTN-CT-2006-035884).

Received: December 24, 2008

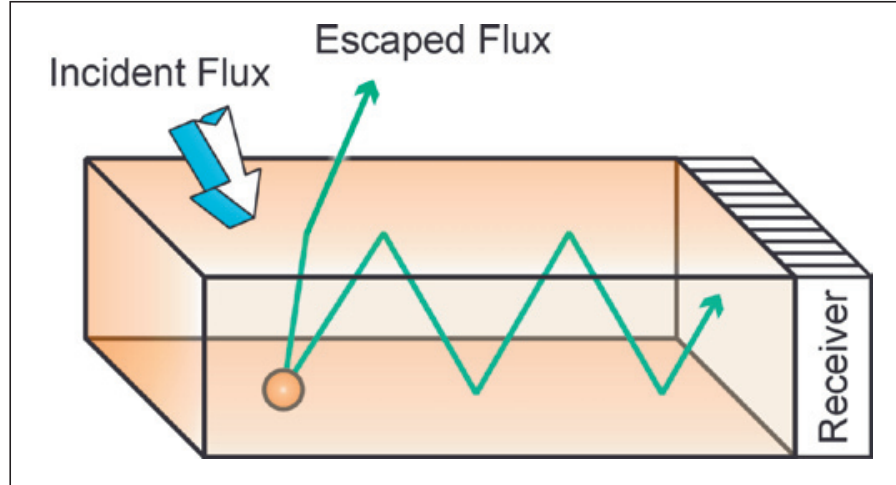

Fig. 8. Working principle of a conventional LSC. If the face area of the plate is larger than its edge area, the device can act as a concentrator of light.
[1] a) A. Corma, Chem. Rev. 1997, 97, 2373; b) A. Corma, J. Catal. 2003, 216, 298; c) A. Taguchi, F. Schüth, Microporous Mesoporous Mater. 2005, 77, 1; d) M. Hartmann, Chem. Mater. 2005, 17, 4577; e) J. H. Clark, D. J. Macquarrie, S. J. Tavener, Dalton Trans. 2006, 4297.

[2] a) M. Tsotsalas, M. Busby, E. Gianolio, S. Aime, L. De Cola, Chem. Mater. 2008, 20, 5888; b) Y. Yang, X. Yan, Y. Cui, Q. He, D. Li, A. Wang, J. Fei, J. Li, J. Mater. Chem. 2008, 18, 5731; c) C.-P. Tsai, Y. Hung, Y.-H. Chou, D.-M. Huang, J.-K. Hsiao, C. Chang, Y.-C. Chen, C.-Y. Mou, Small 2008, 4, 186.

[3] a) N. K. Mal, M. Fujiwara, Y. Tanaka, Nature 2003, 421, 350; b) Q. Yang, S. Wang, P. Fan, L. Wang, Y. Di, K. Lin, F.-S. Xiao, Chem. Mater. 
2005, 17, 5999; c) T. D. Nguyen, Y. Liu, S. Saha, K. C.-F. Leung, J. F. Stoddart, J. I. Zink, J. Am. Chem. Soc. 2007, 129, 626; d) I. I. Slowing, J L. Vivero-Escoto, C.-W. Wu, V. S.-Y. Lin, $A d v$ Drug Deliv. Rev. 2008, 60, 1278.

[4] a) K. A. Fisher, K. D. Huddersman, M. J. Taylor, Chem. Eur. J. 2003, 9, 5873; b) M. Vallet-Regí, F. Balas, D. Arcos, Angew. Chem. Int. Ed. 2007, 46, 7548; c) M. G. Rimoli, M. R. Rabaioli, D Melisi, A. Curcio, S. Mondello, R. Mirabelli, E. Abignente, J. Biomed. Mater. Res. A 2008, 87, 156.

[5] G. Calzaferri, H. Li, D. Brühwiler, Chem. Eur J. 2008, 14, 7442 .

[6] a) C. T. Kresge, M. E. Leonowicz, W. J. Roth, J. C. Vartuli, J. S. Beck, Nature 1992, 359, 710; b) J. S. Beck, J. C. Vartuli, W. J. Roth, M. E. Leonowicz, C. T. Kresge, K. D. Schmitt, C. T.-W. Chu, D. H. Olson, E. W. Sheppard, S. B. McCullen, J. B. Higgins, J. L. Schlenker, J. Am. Chem. Soc. 1992, 114, 10834.

[7] Y. Wan, D. Zhao, Chem. Rev. 2007, 107, 2821.

[8] a) D. Zhao, J. Feng, Q. Huo, N. Melosh, G. H. Fredrickson, B. F. Chmelka, G. D. Stucky, Science 1998, 279, 548; b) D. Zhao, Q. Huo, J. Feng, B. F. Chmelka, G. D. Stucky, J. Am. Chem. Soc. 1998, 120, 6024

[9] F. Hoffmann, M. Cornelius, J. Morell, M. Fröba, Angew. Chem. Int. Ed. 2006, 45, 3216.

[10] H. Ritter, M. Nieminen, M. Karppinen, D. Brühwiler, Microporous Mesoporous Mater, in press.

[11] D. Brühwiler, H. Frei, J. Phys. Chem. B 2003, 107,8547

[12] J. Rouquerol, D. Avnir, C. W. Fairbridge, D. H. Everett, J. H. Haynes, N. Pernicone, J. D. F. Ramsay, K. S. W. Sing, K. K. Unger, Pure Appl. Chem. 1994, 66, 1739.

[13] a) A. Zabala Ruiz, D. Brühwiler, T. Ban, G. Calzaferri, Monatsh. Chem. 2005, 136, 77; b A. Zabala Ruiz, D. Brühwiler, L.-Q. Dieu, G. Calzaferri, in 'Materials Syntheses', Eds. U. Schubert, N. Hüsing, R. Laine, Springer, Wien, 2008, p. 9

[14] I. G. Shenderovich, G. Buntkowsky, A. Schreiber, E. Gedat, S. Sharif, J. Albrecht, N S. Golubev, G. H. Findenegg, H.-H. Limbach, J. Phys. Chem. B 2003, 107, 11924.

[15] C. Barbé, J. Bartlett, L. Kong, K. Finnie, H. Q. Lin, M. Larkin, S. Calleja, A. Bush, G. Calleja, Adv. Mater. 2004, 16, 1959.
[16] T. Yokoi, H. Yoshitake, T. Tatsumi, J. Mater. Chem. 2004, 14, 951.

[17] K. K. Sharma, A. Anan, R. P. Buckley, W. Ouellette, T. Asefa, J. Am. Chem. Soc. 2008, 130, 218.

[18] H. Salmio, D. Brühwiler, J. Phys. Chem. C 2007, 111,923.

[19] D. S. Shephard, W. Zhou, T. Maschmeyer, J. M. Matters, C. L. Roper, S. Parsons, B. F. G. Johnson, M. J. Duer, Angew. Chem. Int. Ed. 1998, 37, 2719.

[20] V. Antochshuk, M. Jaroniec, Chem. Mater. 2000, 12, 2496.

[21] H. Ritter, D. Brühwiler, submitted.

[22] a) M. Weigele, S. L. DeBernardo, J. P. Tengi, W. Leimgruber, J. Am. Chem. Soc. 1972, 94, 5927 ; b) S. Udenfriend, S. Stein, P. Böhlen, W. Dairman, W. Leimgruber, M. Weigele, Science 1972, 178, 871 .

[23] a) Y.-S. Lin, C.-P. Tsai, H.-Y. Huang, C.-T. Kuo, Y. Hung, D.-M. Huang, Y.-C. Chen, C.-Y. Mou, Chem. Mater. 2005, 17, 4570; b) I. Slowing, B. G. Trewyn, V. S.-Y. Lin, J. Am. Chem. Soc. 2006, 128, 14792.

[24] a) D. Brühwiler, G. Calzaferri, Microporous Mesoporous Mater. 2004, 72, 1; b) G. Calzaferri, K. Lutkouskaya, Photochem. Photobiol. Sci. 2008, 7, 879 .

[25] L.-Q. Dieu, A. Devaux, I. Lòpez-Duarte, M. V. Martinez-Diaz, D. Brühwiler, G. Calzaferri, T. Torres, Chem. Commun. 2008, 1187.

[26] D. Brühwiler, L.-Q. Dieu, G. Calzaferri, Chimia 2007, 61, 820.

[27] a) W. H. Weber, J. Lambe, Appl. Opt. 1976, 15, 2299; b) A. Goetzberger, W. Greubel, Appl. Phys. 1977, 14, 123.

[28] a) B. C. Rowan, L. R. Wilson, B. S. Richards, IEEE Journal of Selected Topics in Quantum Electronics 2008, 14, 1312; b) M. J. Currie, J. K. Mapel, T. D. Heidel, S. Goffri, M. A. Baldo, Science 2008, 321, 226.

[29] R. W. Olson, R. F. Loring, M. D. Fayer, Appl. Opt. 1981, 20, 2934.

[30] J. S. Batchelder, A. H. Zewail, T. Cole, Appl. Opt. 1979, 18, 3090.

[31] G. Calzaferri, A. Kunzmann, D. Brühwiler, C. Bauer, Patent pending.

[32] A. Herrmann, K. Müllen, Chem. Lett. 2006, 35, 978. 